\title{
O Peer Instruction (instrução por pares) como estratégia metodológica no ensino-aprendizagem da reanimação neonatal
}

\author{
Peer Instruction as a methodological strategy in the teaching-learning of neonatal \\ resuscitation
}

Peer Instruction (instrucción entre pares) como estrategia metodológica en la enseñanzaaprendizaje de la reanimación neonatal

Edilene Silva da Costa ${ }^{1}$, Elza Ezilda Valente Dantas ${ }^{1}$, Ana Rachel Pinto ${ }^{1}$, Carlos Wagner Machado Pereira ${ }^{2}$.

\section{RESUMO}

Objetivo: Avaliar o nível de aprendizado, satisfação e motivação sobre o curso de reanimação neonatal, comparando as avaliações dos alunos referentes a metodologia tradicional e Peer Instruction (PI). Métodos: Estudo do tipo experimental, abordagem qualiquantitativa realizada com acadêmicos do $5^{\circ}$ ano de medicina, cursando o módulo prático de Pediatria. Os dados foram produzidos com aplicação de questionários com testes de múltipla escolha sobre reanimação neonatal e opiniões sobre as metodologias empregadas. Resultados: Os acertos das questões evidenciaram um nível de aprendizado semelhantes nas duas metodologias durante a primeira rodada de votação, porém na segunda rodada, onde os acertos foram de $30-70 \%$ os alunos conversaram com seus pares e votaram novamente, gerando uma diferença estatisticamente significativa $\left(p=0,0065^{\star}\right)$. O grupo $\mathrm{PI}$ alcançou um desfecho estatisticamente significativo na resposta concordo totalmente $\left(p=0,0325^{\star}\right)$. Os discentes de ambas metodologias discordam, em sua maioria, que haja barreiras de aprendizagem em relação às metodologias aplicadas sendo que o grupo $\mathrm{PI}$ obteve diferença estatisticamente significante na resposta discordo totalmente $\left(p=0.0465^{\star}\right)$. $O$ tema que gerou maior diferença estatística foi a motivação gerada na $\mathrm{PI}\left(p=0,0021^{*}\right)$. Conclusão: $\mathrm{OPI}$ alcançou resultados promissores, confirmando-se como metodologia inovadora, capaz de gerar aprendizagem significativa.

Palavras-chave: Metodologia, Ensino, Reanimação, Motivação.

\begin{abstract}
Objective: Assess the level of learning, satisfaction and motivation about the neonatal resuscitation course, comparing the students' assessments regarding traditional methodology and Peer Instruction (PI). Methods: Experimental type study, qualitative-quantitative approach carried out with 5th year medical students, studying the practical module of Pediatrics. The data were produced using questionnaires with multiple choice tests on neonatal resuscitation and opinions on the methodologies employed. Results: The correctness of the questions showed a similar level of learning in the two methodologies during the first round of voting, however in the second round, where the answers were $30-70 \%$, the students talked to their peers and voted again, generating a statistically significant difference $\left(p=0.0065^{*}\right)$. The PI group achieved a statistically significant outcome in the answer I totally agree $\left(p=0.0325^{*}\right)$. The students of both methodologies disagree, for the most part, that there are learning barriers in relation to the applied methodologies, and the PI group obtained a statistically significant difference in the answer totally disagree $\left(p=0.0465^{*}\right)$. The theme that generated the greatest statistical difference was the motivation generated in IP $\left(p=0.0021^{*}\right)$. Conclusion: The PI achieved promising results, confirming itself as an innovative methodology, capable of generating significant learning.
\end{abstract}

Keywords: Methodology, Teaching, Resuscitation, Motivation.

${ }^{1}$ Centro Universitário do Estado do Pará (CESUPA), Belém - PA. *E-mail: wagnerufpa@gmail.com

2 Universidade Federal do Pará (UFPA), Altamira - PA.

SUBMETIDO EM: 10/2020

ACEITO EM: 10/2020

PUBLICADO EM: 1/2021

REAS/EJCH | Vol.13(1) | e5457 | DOI: https://doi.org/10.25248/reas.e5457.2021 Página 1 de 9 


\section{RESUMEN}

Objetivo: Valorar el nivel de aprendizaje, satisfacción y motivación sobre el curso de reanimación neonatal, comparando las valoraciones de los estudiantes con respecto a la metodología tradicional y Peer Instruction (PI). Métodos: Estudio de tipo experimental, abordaje cualitativo-cuantitativo realizado con estudiantes de $5^{\circ}$ curso de Medicina, cursando el módulo práctico de Pediatría. Los datos se elaboraron mediante cuestionarios con pruebas de opción múltiple sobre reanimación neonatal y opiniones sobre las metodologías empleadas. Resultados: La veracidad de las preguntas mostró un nivel de aprendizaje similar en las dos metodologías durante la primera ronda de votación, sin embargo en la segunda ronda, donde las respuestas fueron 30 $70 \%$, los estudiantes hablaron con sus compañeros y votaron nuevamente, generando una diferencia estadísticamente significativa ( $\left.p=0,0065^{*}\right)$. El grupo de IP logró un resultado estadísticamente significativo en la respuesta Estoy totalmente de acuerdo $(p=0.0325 *)$. Los estudiantes de ambas metodologías discrepan, en su mayor parte, de que existen barreras de aprendizaje en relación a las metodologías aplicadas, y el grupo de IP obtuvo una diferencia estadísticamente significativa en la respuesta totalmente en desacuerdo $(p=0.0465 *)$. El tema que generó mayor diferencia estadística fue la motivación generada en PI $\left(p=0,0021^{*}\right)$. Conclusión: El IP logró resultados prometedores, confirmándose como una metodología innovadora, capaz de generar aprendizajes significativos.

Palabras clave: Metodología, Docencia, Reanimación, Motivación.

\section{INTRODUÇÃO}

Historicamente, a formação dos profissionais da saúde tem sido baseada no uso de metodologias tradicionais, direcionada pelo mecanismo de inspiração Cartesiano-Newtoniana, fazendo com que o processo ensino-aprendizagem se restrinja a reprodução do conhecimento, muitas vezes cansativa, no qual o professor assume um papel de transmissor de conteúdos e o discente se comporta de forma passiva, sem a consciência crítica, indagadora, criativa e insatisfeita de um indivíduo ativo dentro do processo de aprendizagem (MITRE SM, et al., 2014).

Godoi AF e Ferreira JV (2016) discutem sobre a percepção das diversas mudanças no cenário médico e cientifico, as faculdades perceberam a necessidade de modificação das grades curriculares para que os alunos obtivessem durante seu período de formação a solidez e segurança necessárias para a atuação profissional. A introdução de cadeiras básicas, com a construção de laboratórios, possibilitou aos professores a atuação simultânea na pesquisa cientifica e docência.

Essas mudanças propiciaram o contato dos alunos com a realidade prática, tema amplamente discutido no âmbito educacional, pois a partir das novas diretrizes, os alunos estudariam os pacientes dentro dos Hospitais Universitários, sob a tutela de professores experientes e renomados na ciência e docência médica.

Neste sentido, o aperfeiçoamento de metodologias de instrução no ensino superior é trazido com mais força por pesquisadores em diferentes segmentos da ciência, sendo a Andragogia, o principal caminho na orientação e aprendizagem de adultos, ganhando reconhecimento na literatura da educação, estando presente em metodologias ativas tais como o Peer Instruction (Instrução por Pares), sendo esta uma alternativa às metodologias tradicionais (GODOI AF e FERREIRA JV, 2016).

Vygotsky LS (2012) defende que as evoluções pedagógicas e metodológicas emergem para as novas realidades sociais em decorrência das mudanças na práxis médica, tornando indispensável a admissão de modernas formas de ensinar e aprender no ensino médico. Isto pode ser confirmado pelas constantes modificações presentes na educação, sendo o uso de tecnologias essencial para que a dinâmica em sala de aula torne-se interessante para o aluno e produtiva para o professor.

Anderson T e Dron J (2011) dizem que o uso de mecanismos online (redes sociais) propicia um modo adicional de interação entre o aluno e o professor, aproximando-os e possibilitando um contato sócio construtivista. 
Villas-Boas V e Mattasoglio NO (2011) esclarecem que o Peer instruction (PI) se estrutura da seguinte maneira: Primeiro, o professor apresenta uma questão qualitativa e normalmente em formato de múltipla escolha, sendo necessário que o professor pense a questão de acordo com as dificuldades dos alunos. Posteriormente, os alunos devem votar, habitualmente por meio eletrônico, sendo importante ressaltar que é possível identificar a fração da turma que dá a resposta, podendo ser relatada pelo docente.

Após a votação, os alunos discutem o problema apresentado pelo professor por cerca de dois minutos, com o intuito de convencer seus pares do motivo da resposta, apontado porque tal posicionamento é o correto, e por fim, uma nova votação é realizada, permitindo que ao final o professor abra espaço para perguntas e discussões sobre as possíveis respostas e a solução de fato da questão apresentada.

Araújo IS e Mazur E (2013) corroboram com esse pensamento afirmando que o PI é uma estratégia de ensino criada para que os alunos sejam intelectualmente estimulados a interagir de forma ativa com o conteúdo ministrado em sala de aula, assim sendo o PI evidencia-se pelo entendimento e aplicabilidade dos conceitos, gerando uma discussão entre os discentes e relatam que metodologias ativas de ensino ainda são pouco difundidas e utilizadas no Brasil, sendo desconhecidas por grande parte dos professores.

Fato que se mostra preocupante, logo que diversas pesquisas demonstram a grande melhora no aprendizado de alunos submetidos ao ensino através de metodologias ativas. A aplicação da metodologia PI prevê o uso de tecnologias como, por exemplo, o uso de gerenciamento de respostas que são dispositivos individuais fornecidos aos alunos que participam desta metodologia como, por exemplo, plataformas online como o aplicativo Socrative, além de fichas, folhas ou filipetas de votação, cartões ou a indicação pelo aluno com a mão para sinalizar a resposta correta das questões formuladas.

Bezerra AAC (2016) relatam que o Socrative é um software gratuito, que se encontra disponível na internet e em lojas online para dispositivos móveis, tendo como funcionalidade o ensino e o aprendizado através de um celular ou tablete em sala de aula e mudou a vivencia e participação dos alunos e docentes em sala de aula, promovendo a reflexão aprofundada sobre a resposta enviada pelos alunos.

Vettori M e Zaro MA (2017) expõem uma experiência avaliando o Socrative e a metodologia PI, tendo sido aplicada em discentes de engenharia em uma cadeira de física geral, ocasião em que foi possível perceber um maior engajamento dos discentes a partir das atividades ofertadas no aplicativo, que aliado à metodologia ativa, produziram efeitos positivos, uma aprendizagem significativa nos alunos e esclarecem que a associação do PI ao software em questão se mostra uma importante ferramenta para o processo de aprendizagem ativa, possibilitando um feedback mais rápido ao docente sobre o processo de aprendizagem.

Belloni ML (2015) comenta que a grande dificuldade do ensino de nível superior é conectar a prática profissional, relacionada aos problemas habituais com os conceitos acadêmicos estudados nas salas de aula. $\mathrm{Em}$ todas as formações profissionais, acredita-se que as universidades possam estimular o desenvolvimento das competências discentes, contemplando não somente o conhecimento, através de temas específicos de cada área, mas também no que se refere a condutas e habilidades, que apontam para os vários tipos de saberes que são utilizados na solução dos problemas do cotidiano. Assim, a metodologia do PI envolve, compromete e mantém os alunos atentos durante a aula por meio de dinâmicas que exigem a aplicação dos conceitos fundamentais apresentados pelo docente, e, em seguida, sua explicação para os demais colegas.

No Brasil, dados do Ministério da Saúde revelam que a mortalidade neonatal é responsável por cerca de $70 \%$ das mortes no primeiro ano de vida. Nesse sentido observa-se a necessidade de uma adequada atenção ao recém-nascido (RN) como um dos importantes desafios para diminuir os índices de morbimortalidade infantil. Visando mudar essa realidade, em 2011 o Ministério da Saúde lançou no Brasil a Rede Cegonha que tem como objetivos assegurar às crianças nascimento e crescimento seguro e saudável, proporcionando para todos os RNs boas práticas de atenção baseadas em evidências científicas e em princípios de humanização.

Considerando que aproximadamente 1 em cada $10 \mathrm{RNs}$ necessitará de alguma ajuda para respirar, sendo que cerca de $1 \%$ precisará de esforços intensivos de reanimação, mostrando que a equipe de sala de parto deve estar apta a fornecer os cuidados necessários, uma vez que no Brasil nascem, por ano, aproximadamente 3 milhões de crianças. Nessa ótica, deve-se ressaltar que o número absoluto dos RNs que necessitarão de algum grau de reanimação acaba se tornando alto (BRASIL, 2017). 
Atualmente o PRN já treinou milhares de profissionais em todos os estados do País. Segundo o último levantamento feito pela coordenação do Programa, desde a implantação das condutas atualizadas de 2016, já foram certificados mais de 16 mil médicos e nove mil profissionais de saúde no curso de Reanimação Neonatal (MITCHELL A, et al., 2018).

O curso de Reanimação do Recém-nascido em sala de parto da Sociedade Brasileira de Pediatria, disponível para médicos e acadêmicos de medicina a partir do quinto ano, é realizado em oito horas, sendo duas horas de aulas teóricas no estilo tradicional, nas quais o aluno se comporta de forma passiva, gerando desmotivação e desinteresse (ALMEIDA MFB e GUINSBURG R, 2016).

O treinamento em reanimação neonatal constitui-se em estratégia relativamente simples e não onerosa para diminuir a mortalidade neonatal precoce. Dentre as várias maneiras de aperfeiçoar o conhecimento dos profissionais no tema, a mais bem sucedida é o PRN (Programa de Reanimação Neonatal), figurando como uma proposta do Comitê Internacional de Reanimação Neonatal.

O PRN é um curso que permite mudanças na competência profissional, propiciando ganhos de conhecimentos e habilidades técnicas necessárias para a assistência ao recém-nascido, além de possibilitar uma melhora na autoconfiança do médico durante a assistência em sala de parto (BRASIL, 2017).

Partindo desse cenário, foi proposto um estudo que avaliasse o uso de uma metodologia específica no processo de ensino aprendizagem da reanimação neonatal com a inserção do PI como estratégia metodológica inovadora para o curso do Programa de Reanimação Neonatal, buscando comprometimento, trabalho em equipe, comunicação, motivação, aprendizagem significativa, como formas de contribuição acadêmica e pessoal.

\section{MÉTODOS}

Trata-se de um estudo do tipo experimental, com abordagem qualiquantitativa, desenvolvido com acadêmicos do 5o ano de Medicina cursando o módulo prático de Pediatria em um hospital escola no município do interior do Pará em suas atividades práticas no período de março a agosto de 2019.

Na primeira etapa do estudo o pesquisador se reuniu com as turmas, no hospital escola, e foi explicado aos estudantes o propósito da pesquisa, em seguida foi distribuído o Termo de Consentimento Livre e Esclarecido, sendo solicitado a leitura do mesmo pelos discentes.

Após a leitura, o experimentador aguardara alguns minutos para que os estudantes pudessem assinar 0 Termo de Consentimento Livre e Esclarecido e realizar qualquer questionamento sobre eventuais etapas da pesquisa. Enfatiza-se que esta pesquisa atende a resolução n466/ 2012 do Conselho Nacional de Saúde, sendo sido submetido ao comitê de ética em pesquisa em seres humanos do Centro Universitário do Estado do Pará (CESUPA) cujo parecer de aprovação (CAAE 08052418.4.0000.5169).

Em uma segunda etapa, as quatro turmas foram randomizadas através de sorteio realizado pelos próprios estudantes, onde duas turmas receberam capacitação baseada no ensino teórico sobre reanimação neonatal $>34$ semanas com metodologia tradicional (modelo atual), e as outras duas consideradas como experimentais, as mesmas foram submetidas à capacitação com metodologia ativa PI.

Eles foram avaliados pelo teste do curso de Reanimação Neonatal do Programa Brasileira de Pediatria, com questões de múltipla escolha (capacitação Tradicional), e as turmas PI foram avaliados com as mesmas questões, votando online, através do aplicativo Socrative.

O tempo foi limitado, sendo que as turmas da prova tradicional tiveram 50 minutos para responderem 25 questões, média de 2 minutos por questão e as turmas PI tiveram 2 minutos para votarem na questão correta, as questões eram as mesmas da turma tradicional, todas baseadas no teste do curso oficial de Reanimação Neonatal, após a avaliação os sujeitos da pesquisa responderam a um questionário baseado na escala Likert sobre a metodologia utilizada, o resultado das avaliações foi informado aos participantes durante a capacitação, PI de forma instantânea através do aplicativo Socrative e tradicional, logo após a correção das provas. 
As informações coletadas foram inseridas em planilha para banco de dados, as tabelas e gráficos foram construídos no Microsoft EXCEL 2010 e feitas análises da significância com os testes Qui-quadrado Aderência para as tabelas uni-variadas; Qui-quadrado - Independência para as tabelas bivariadas e o Teste $G$ para tabelas com frequência, em alguma categoria, abaixo de 5 (cinco) e Teste t-Student Independente $O$ nível de significância adotado foi $\alpha=0,05(5 \%)$ e os testes estatísticos aplicados através do Software SPSS, assinalando com asterisco $\left({ }^{*}\right)$ os valores significantes.

\section{RESULTADOS E DISCUSSÃO}

Um debate de grande relevância ao estudo pode ser verificado quanto a utilização do método PI, pois esse possibilita que os alunos alcancem um maior nível de motivação, relacionando-se de maneira positiva com os seus pares, o que gera uma aprendizagem cooperativa e significativa.

Isto se confirma porque a metodologia ativa promove uma melhor organização das estruturas do pensamento, trabalhando aptidões como: percepção, análise, estratégia, decisão, planificação e comunicação, diminuindo as barreiras na aquisição do conhecimento (AQUINO FGF, et al., 2015).

A aprendizagem, desde tempos antigos, vem motivando muitos debates. Sobre ela, vários educadores têm desenvolvido, várias teorias. O objetivo do artigo é conhecer as propostas sobre a aprendizagem escolar e a instrução, através de uma teoria atual proposta pelo psicólogo norte-americano David Ausubel (BERWANGER PM, 2018).

É a teoria da aprendizagem significativa, a qual determina que os conhecimentos prévios dos alunos sejam valorizados para que seja possível construir estruturas mentais, usando como recurso, mapas conceituais que possibilitam descobrir ou redescobrir outros conhecimentos, tornando assim, a aprendizagem, satisfatória e prazerosa (FERMOZELLI JA, et al., 2017).

No que se refere a motivação referenciada foi encontrada diferença estatisticamente significante $\left(p=0.0021^{*}\right)$ na avaliação dos discentes em relação a motivação com as metodologias utilizadas. Os discentes que utilizaram a metodologia $\mathrm{PI}$ alcançaram $89.3 \%$ na opção "Concordo totalmente" e, àqueles que utilizaram a metodologia tradicional, ficaram com $40.0 \%$ (Tabela 1 ).

Tabela 1 - Avaliação da motivação gerada pelas metodologias nos alunos.

\begin{tabular}{ccc}
\hline Você se sentiu motivado com a metodologia & $\begin{array}{c}\text { Tradicional (\%) } \\
\mathbf{( N = 3 0 )}\end{array}$ & $\begin{array}{c}\mathbf{P l}(\%) \\
\mathbf{( N = 2 8 )}\end{array}$ \\
\hline Concordo totalmente & 40.0 & 89.3 \\
Concordo parcialmente & 43.3 & 3.6 \\
Indiferente & 3.3 & 7.1 \\
Discordo parcialmente & 10.0 & - \\
Discordo totalmente & 3.3 & - \\
\hline
\end{tabular}

Legenda: ${ }^{*} p=0.021$ Teste $G$ Independência.

Fonte: COSTA ES, et al., 2020.

O processo, através do qual a informação nova interage com a estrutura do conhecimento específico é o chamado conceito "subsunçor". No entanto, quando o conteúdo escolar a aprender não está ligado a algum assunto já conhecido, segundo o autor, acontece a aprendizagem mecânica e, consequentemente, a decoração, que não interage com conceitos importantes que existem na estrutura cognitiva (AQUINO FGF, et al., 2015).

Brito CAF e Campos MZ (2019) apresentaram resultados semelhantes acerca da motivação foram evidenciados no trabalho de durante o emprego de PI nas aulas de Educação física. A compreensão das narrativas dos discentes corroboram com o entendimento dos alunos em participar nas aulas, pois eles descrevem um estado de felicidade; empenho em estudar e se dedicar aos estudos, atribuindo assim a responsabilidade do discente nesta ação-reflexão-ação, bem como satisfação de forma geral (em torno de $63,6 \%)$. 
A votação pela internet despertou interesse de uma proporção (82.1\%) estatisticamente significante $\left(p=0.0001^{*}\right)$ da amostra que utilizou o método PI (Tabela 2).

Tabela 2 - Opinião sobre a votação na internet.

\begin{tabular}{ccc}
\hline Votar por meio da internet foi positivo & PI & (N=28) \\
\hline Concordo totalmente $^{*}$ & 23 & $82.1 \%$ \\
Concordo parcialmente & 3 & $10.7 \%$ \\
Indiferente & - & - \\
Discordo parcialmente & 1 & $3.6 \%$ \\
Discordo totalmente & 1 & $3.6 \%$ \\
\hline
\end{tabular}

Legenda: ${ }^{*} p=0.0001$ Teste G Independência. Fonte: COSTA ES, et al., 2020.

Berwanger PM (2018), nessa ótica, corrobora com esses resultados relatando algumas pesquisas empíricas que abordam os uso do smartphone em contextos educativos no modelo presencial e traz reflexões sobre situações de uso do smartphones articulados a outras tecnologias digitais móveis, porém, não abordam atividades de "avaliação de aprendizagem de conteúdo" que tenham sido realizadas com esses recursos tecnológicos.

Silva SL (2019) acredita que os investimentos em métodos colaborativos são essenciais no itinerário formativo do século $\mathrm{XXI}$, que coloca em evidência que a formação não é mais centrada apenas no componente conceitual, mas a aprendizagem deve ser concebida de forma alargada, na qual a experiência do aluno, o exercício de habilidades como comunicar-se bem, cooperar, debater, geram competências argumentativas e contribuem para uma formação mais integral e que responde aos desafios no novo século e pressupõe uma formação por competências técnicas e transversais, que sejam desenvolvidas por meio de experiências que movimentem os aprendizes a elaborarem e reelaborarem os conceitos articulando-os por meio do pensamento crítico, do engajamento social e da criatividade.

Em relação ao nível de aprendizado após a interação com pares, observou-se uma diferença estatisticamente significante $\left(p=0.0065^{\star}\right)$ quando comparados os resultados da metodologia tradicional com a segunda rodada de votação do grupo que utilizou a metodologia PI (85.1\% - 91.3\%) (Tabela 3).

Tabela 3 - Resultados das avaliações realizadas - nível de aprendizado.

\begin{tabular}{|c|c|c|c|c|c|}
\hline \multirow{2}{*}{ Avaliações realizadas } & \multicolumn{4}{|c|}{ Método utilizado } & \multirow{2}{*}{ p-valor } \\
\hline & \multicolumn{2}{|c|}{ Tradicional } & \multicolumn{2}{|c|}{$\mathbf{P I}$} & \\
\hline Avaliação 1 & $85.1 \%$ & $\pm 14.2 \%$ & $84.2 \%$ & $\pm 15.5 \%$ & 0.3746 \\
\hline Avaliação 2 & ---- & ----- & $91.3 \%$ & $\pm 9.4 \%$ & $0.0065^{*}$ \\
\hline
\end{tabular}

Legenda: *Teste t-Student Independente. Fonte: Costa ES, et al., 2020.

Conceição CV e Moraes MAA (2018) somam seu estudo a esse pensamento com base no entendimento de docentes e estudantes, concluiu-se que a aprendizagem cooperativa é eficaz em pequenos grupos e que traz benefícios sociais, cognitivos e psicomotores, sendo favorecida no âmbito de metodologias ativas e com a educação permanente, a qual, se implementada de maneira incisiva, pode gerar ferramentas úteis ao processo de ensino e aprendizagem.

Esta possibilita ainda, o que na aprendizagem significativa de Ausubel, é a possibilidade de "dar significado a um novo conhecimento, seja de forma mediada, seja pela própria inferência do sujeito, ou seja, aqui a mediação dos pares favorecido pela metodologia do PI, implica numa aprendizagem com mais significado." (MOREIRA MA, 2010).

Versteeg M, et al. (2019) na Universidade de Leiden- Holanda com estudantes de medicina do primeiro ano, n:317, avaliando a compreensão dos conceitos fisiológicos entre PI e a metodologia de auto explicação, sendo que as pontuações pós testes foram significativamente melhoradas $(p<0,0001)$ com o $\mathrm{Pl}$, superando a auto explicação. 
Curiosamente um grande número de alunos com respostas iniciais incorretas, obtiveram êxito após discussão com os colegas. Esses achados apoiam o PI como um método de ensino-aprendizagem valioso para aprimorar a compreensão de conceitos fisiológicos pelos estudantes, novas ideias que confirmam as teorias educacionais ativas fundamentais na busca de uma aprendizagem significativa.

Em outros estudos como o de Petersen MW, et al. (2014), que focaram a aprendizagem do diagnóstico de desordens ácido-base através da análise de gás do sangue arterial. Nesse estudo, houve resultados positivos tanto para o grupo experimental (PI) como para o grupo de controle (atividades colaborativas em grupo), revelando que ambas as metodologias podem contribuir para a aprendizagem dos estudantes e assim demonstrar que toda forma de conhecimento é agrega de forma contribuidora ao processo de ensinoaprendizagem.

Sendo assim, observamos que os discentes concordam que as utilizações de ambas as metodologias são positivas nas aulas, não havendo diferença estatisticamente significante $(p=0.8564)$, entre as opiniões dos grupos (Tabela 4).

Tabela 4 - Avaliação positiva em relação à metodologia utilizada nas aulas.

\begin{tabular}{ccccc}
\hline \multirow{2}{*}{ A utilização da metodologia foi positiva nas aulas? } & \multicolumn{2}{c}{ Tradicional } & \multicolumn{2}{c}{$\mathbf{P I}$} \\
\cline { 2 - 5 } & $\mathbf{( N = 3 0 )}$ & $\mathbf{( \% )}$ & $\mathbf{( N = 2 8 )}$ & $\mathbf{( \% )}$ \\
\hline Concordo totalmente & 22 & 73.3 & 20 & 71.4 \\
Concordo parcialmente & 7 & 23.3 & 7 & 25.0 \\
Indiferente & 1 & 3.3 & 1 & 3.6 \\
Discordo parcialmente & 1 & 3.3 & - & - \\
\hline
\end{tabular}

Legenda: ${ }^{*} p=0.8564$ Teste G Independência. Fonte: COSTA ES, et al., 2020.

Estudos como de Lasry N (2018) apontam que a adoção do PI pode contribuir para uma maior adesão dos alunos de forma ativa no processo de ensino aprendizagem. Neste estudo se buscou analisar o número de estudantes que finalizaram a disciplina em que aplicaram o $\mathrm{PI}$, comparativamente a turmas com aulas tradicionais e um baixo índice não finalizou o curso. Em contraste, o índice de estudantes que desistiu do curso na turma experimental maior de $20 \%$. Logo, reforçando que a utilização de metodologias ativas garante maior adesão do alunado.

Foi observado que os discentes divergiram de forma estatisticamente significante $\left(p=0.0325^{\star}\right)$ em suas opiniões, em relação à vantagem das metodologias comparadas a outras. Os discentes da metodologia $\mathrm{PI}$ alcançaram maior proporção na opção "Concordo totalmente" (53.6\%), enquanto os discentes da metodologia Tradicional obtiveram seu maior percentual, na opção "Concordo parcialmente" (60.0\%) (Tabela 5).

Tabela 5 - Avaliação da vantagem do uso da metodologia PI na capacitação realizada em um município do interior do Pará, 2019.

\begin{tabular}{cccccc}
\hline \multirow{2}{*}{ A Metodologia PI foi vantajosa quando comparada as demais } & \multicolumn{3}{c}{ Tradicional } & \multicolumn{3}{c}{$\mathbf{P I}$} \\
& $\mathbf{( N = 3 0 )}$ & $\mathbf{( \% )}$ & $\mathbf{( N = 2 8 )}$ & (\%) \\
\hline Concordo totalmente & 7 & 23.3 & 15 & 53.6 \\
Concordo parcialmente & 18 & 60.0 & 11 & 39.3 \\
Indiferente & 3 & 10.0 & - & - \\
Discordo parcialmente & 2 & 6.7 & 2 & 7.1 \\
Discordo totalmente & - & - & - & - \\
\hline
\end{tabular}

Legenda: ${ }^{*} p=0.0325$ Teste G Independência. Fonte: Costa ES, et al., 2020.

Outro ponto observável de discordância, entre os discentes de ambas as metodologias, em sua maioria, é que haja barreiras de aprendizagem em relação às metodologias aplicadas, sendo que os discentes do método PI alcançaram diferença estatisticamente significante $\left(p=0.0465^{*}\right)$ em suas proporções, em relação ao método tradicional $(60.0 \%-40.0 \%$ respectivamente) (Tabela 6$)$. 
Este resultado pode ser verificado pois o método PI possibilita que os alunos alcancem um maior nível de motivação e diminuam suas barreiras de aprendizagem, relacionando-se de maneira positiva com os seus pares, o que gera uma aprendizagem cooperativa e significativa. Isto se confirma porque a metodologia ativa promove uma melhor organização das estruturas do pensamento, trabalhando aptidões como: percepção, análise, estratégia, decisão, planificação e comunicação, diminuindo as barreiras na aquisição do conhecimento (LASRY N, 2018).

Tabela 6 - Avaliação sobre a ocorrência de barreiras de aprendizagem na capacitação realizada em Marituba, Pará, 2019.

\begin{tabular}{ccccc}
\hline \multirow{2}{*}{ Houve barreiras de aprendizagem em relação à metodologia } & \multicolumn{2}{c}{ Tradicional } & \multicolumn{2}{c}{$\mathbf{P I}$} \\
\cline { 2 - 5 } & $\mathbf{( N = 3 0 )}$ & $\mathbf{( \% )}$ & $\mathbf{( N = 2 8 )}$ & $\mathbf{( \% )}$ \\
\hline Concordo totalmente & 1 & 3.3 & - & - \\
Concordo parcialmente & 6 & 20.0 & 1 & 3.6 \\
Indiferente & 5 & 16.7 & 1 & 3.6 \\
Discordo parcialmente & 6 & 20.0 & 9 & 32.1 \\
Discordo totalmente & 12 & 40.0 & 17 & 60.7 \\
\hline
\end{tabular}

Legenda: ${ }^{*} p=0.0465$ Teste G Independência. Fonte: COSTA ES, et al., 2020.

\section{CONCLUSÃO}

Ao analisar os resultados observamos um nível de aprendizagem melhor após a segunda rodada de votação, em que os pares conversam entre si, mostrando a essência da metodologia empregada, instrução por pares, e ratificando a importância de construir metodologia ativa. Além disso, a experiência educativa mostra que há uma vantagem significativa do PI quando comparadas a outras metodologias quanto a uma melhor sedimentação do Programa de Reanimação Neonatal. Tornando-a uma ferramenta cada vez mais efetiva e de baixo custo na redução da mortalidade infantil. O PI alcançou resultados promissores, confirmando-se como metodologia inovadora, capaz de gerar aprendizagem significativa e conhecimento multiplicador.

\section{REFERÊNCIAS}

1. ALMEIDA MFB, et al. Ensino da reanimação neonatal em maternidades públicas das capitais brasileiras. Jornal de Pediatria, 2015; 81(3): 233-239.

2. ANDERSON T, DRON J. Three generations of distance education pedagogy. The International Review of Research in Open and Distributed Learning, 2011; 12(3).

3. AQUINO FGF, et al. AUSUBEL: Aprendizagem significativa e avaliação. Revista Atlante: Cuadernos de Educación y Desarrollo, 2015; 5(8): 13-20.

4. ARAÚJO IS, MAZUR E. Instrução pelos colegas e ensino sob medida: uma proposta para o engajamento dos alunos no processo de ensino-aprendizagem de física. Caderno Brasileiro de Ensino de Física, 2013; 30(2): $362-384$.

5. BELLONI ML. O que é mídia educação? 2. ed. Campinas, SP, 2015.

6. BERWANGER PM. Aprender e ensinar na era digital: um estudo sobre mobile learning em experiências de avaliação de aprendizagem na educação superior um estudo sobre mobile learning em experiências de avaliação de aprendizagem na educação superior. Dissertação (Mestrado em Educação) - Instituto de Letras e Artes, Universidade Federal do Maranhão, São Luís, 2018; 194 p.

7. BEZERRA AAC. Socrative: O amigo do professor. Encontro Internacional de Formação de Educadores, Aracaju, 2018; 3(40): 235-300.

8. BRASIL. Ministério da Saúde. Prevenção à mortalidade infantil. Brasília, DF, 2017.

9. BRITO CAF, CAMPOS MZ. Facilitando o processo de aprendizagem no ensino superior: o papel das metodologias ativas. Revista Ibero-americana de Estudos em Educação, 2019; 142: 01-34.

10. CONCEIÇÃO CV, MORAES MAA. Aprendizagem cooperativa e a formação do médico inserido em metodologias ativas: um olhar de estudantes e docentes. Revista Brasileira de Educação Médica, 2018; 42(4): 115-122.

11. FERMOZELLI JÁ, et al. Blended learning strategies in teaching general pathology at a medical course. Jornal Brasileiro de Patologia e Medicina Laboratorial, 2017; 53(3): 300-345.

12. GODOI AF, FERREIRA JV. Metodologia ativa de aprendizagem para o ensino em administração: relatos da experiência com a aplicação do peer instruction em uma instituição de ensino superior. Revista Eletrônica de Administração, 2016; 15(2)

13. LASRY N. Clickers or flashcards: is there really a difference?. The Physics Teach, 2018; 46(2). 
14. MITCHELL A, et al. A prospective clinical audit of neonatal resuscitation practices in Canada. Adv Neonatal Care, 2018; 2: 316-326.

15. MITRE SM, et al. Metodologias ativas de ensino e aprendizagem na formação profissional: debates atuais. Ciência Saúde Coletiva, 2014; 13(2): 2133-2144.

16. Moreira, M.A. Mapas conceituais e aprendizagem significativa. Centauro Editora, 2018; 15: 80.

17. PETERSEN MW, et al. AJP: Advances in physiology education, 2014; 38: 101.

18. RIBEIRO MAS, LOPES MHI. Impacto do programa de reanimação neonatal. Scientia Medica, 2017; $17(2): 79-86$.

19. SILVA SL. Ensino de bioética na graduação de medicina: relato de experiência. Rev. Bioética, Recife, PE, 2019; 21(2): 338-43.

20. VERSTEEG M, et al. Peer instruction improves comprehension and transfer of physiological concepts: a randomized comparison with self-explanation. Lieden Advances in Health Sciences Education, 2019; 24: 151-165.

21. VETTORI M, ZARO MA. Avaliação do socrative app como ferramenta auxiliar de ensino para a construção de aprendizagens significativas em uma disciplina de física geral a partir do peer instruction. Anais dos Workshops do IV Congresso Brasileiro de Informática na Educação (CBIE), 2016.

22. VILLAS-BOAS V, MATTASOGLIO NO. Aprendizagem ativa na educação em engenharia. Congresso Brasileiro de Educação em Engenharia, Blumenau, 2011.

23. VYGOTSKY LS. Thought and language. Cambridge, MA: MIT Press, 2012. 